\title{
Mapping of Dissipative Particle Dynamics in Fluctuating Hydrodynamics Simulations
}

\author{
R. Qiad* and P. He \\ College of Engineering \& Sciences, Clemson University, Clemson, SC, 29634
}

(Dated: August 18, 2021)

\begin{abstract}
Dissipative particle dynamics (DPD) is a novel particle method for mesoscale modeling of complex fluids. DPD particles are often thought to represent packets of real atoms, and the physical scale probed in DPD models are determined by the mapping of DPD variables to the corresponding physical quantities. However, the non-uniqueness of such mapping has led to difficulties in setting up simulations to mimic real systems and in interpreting results. For modeling transport phenomena where thermal fluctuations are important (e.g., fluctuating hydrodynamics), an area particularly suited for DPD method, we propose that DPD fluid particles should be viewed as only 1) to provide a medium in which the momentum and energy are transferred according to the hydrodynamic laws and 2) to provide objects immersed in the DPD fluids the proper random "kicks" such that these objects exhibit correct fluctuation behaviors at the macroscopic scale. We show that, in such a case, the choice of system temperature and mapping of DPD scales to physical scales are uniquely determined by the level of coarse-graining and properties of DPD fluids. We also verified that DPD simulation can reproduce the macroscopic effects of thermal fluctuation in particulate suspension by showing that the Brownian diffusion of solid particles can be computed in DPD simulations with good accuracy.
\end{abstract}

Dissipative particle dynamics (DPD) is a method developed primarily for the simulation of complex fluids at mesoscopic scales $\frac{1,2,3}{2}$. While it is often thought that DPD beads represent packets of real atoms moving collectively, the statistical mechanical foundation of such a view for DPD model remains obscure ${ }^{4}$. Associated with the ambiguity of the exact nature of DPD beads is the ambiguity in mapping of DPD scales to the physical scales. While the mapping of length is straightforward, the mapping of time is more complicated. Depending on the problems being studied, mapping of DPD time to physical time has been based on bead diffusion rate, bead thermal velocity, or externally imposed time scales ${ }^{5.6,7}$, to name just a few. In all but a few cases $^{7}$, temperature of the DPD system was chosen arbitrarily. Established procedures for mapping between DPD and physical scales and for choosing system temperature are not yet available.

Our interest in DPD originates from the need to study transport phenomena in particulate suspension where thermal fluctuations may play a critical role ${ }^{8.9}$. Such transport can be described by the fluctuating hydrodynamics theories $\underline{10}$. However, solving the hydrodynamics equations in particulate suspension is computationally demanding and introducing thermal fluctuations that rigorously satisfy the Fluctuation-Dissipation Theorem (FDT) is challenging 11 . DPD is well-suited for studying such phenomena as momentum/energy conservation and FDT are guaranteed by the way DPD models are designed, and colloidal particles can be modeled eas-

*Corresponding author. Email: rqiao@ces.clemson.edu ily by bonding DPD beads together ${ }^{2.3}$. However, there are two unresolved issues in DPD simulation of fluctuating hydrodynamics, namely, how to set up a model for a given physical system and how to interpret the simulation results. For example, to study the diffusion of a $30 \mathrm{~nm}$ diameter particle in water at $300 \mathrm{~K}$, what should be the temperature of the DPD system and how to map the results to dimensional values are not clear.

To address the above issues, we propose to abandon the idea that DPD fluid beads are "clumps" of real fluid atoms, but view them together as a "media" that provides an arena for the transport of momentum, energy and particulates that satisfies the fluctuating hydrodynamics laws. This idea is inspired by a recent paper on coarse-graining in colloidal suspensions ${ }^{4}$. To bridge the DPD and physical systems, we require that

1. The time scale of diffusional transport of momentum (and energy if in non-isothermal simulations) inside the DPD fluids should match that of the real fluids;

2. DPD fluids should provide objects immersed in them the proper random "kicks" such that these objects exhibit correct fluctuation behaviors at the macroscopic scale.

We now consider the application of the above requirements in the simulation of colloidal particles (diameter: $\bar{d}$ ) dispersed in fluids (density: $\bar{\rho}$, kinematic viscosity: $\bar{\nu}$, temperature: $\bar{T}$ ). Properties of corresponding DPD fluids are denoted by the same symbol as in real fluids but without the bar, e.g., density of DPD fluids is denoted as $\rho$. We will limit 
our discussion to isothermal simulations, and the extension to non-isothermal simulations is straightforward. The DPD model reads $s^{3}$

$$
d \boldsymbol{r}_{i}=\boldsymbol{v}_{i} d t ; \quad m d \boldsymbol{v}_{i}=\boldsymbol{F}_{i}^{C} d t+\boldsymbol{F}_{i}^{D} d t+\boldsymbol{F}_{i}^{R} \sqrt{d t}
$$

where $m, \boldsymbol{r}_{i}$, and $\boldsymbol{v}_{i}$ are the mass, position, and velocity of bead $i$, respectively. $T$ is the system temperature. $\mathbf{F}_{i}^{C}, \mathbf{F}_{i}^{D}$ and $\mathbf{F}_{i}^{R}$ are the conservative, dissipative and random forces acting on bead $i$, respectively. These forces are given by $\underline{\underline{3}, 12}$

$$
\begin{aligned}
\boldsymbol{F}_{i}^{C} & =\sum_{j \neq i} a_{i j} w\left(r_{i j} / r_{c}\right) \boldsymbol{e}_{i j} \\
\boldsymbol{F}_{i}^{D} & =\sum_{j \neq i}-\gamma_{i j} w_{d}^{2}\left(r_{i j} / r_{c}^{d}\right)\left(\boldsymbol{e}_{i j} \cdot \boldsymbol{v}_{i j}\right) \boldsymbol{e}_{i j} \\
\boldsymbol{F}_{i}^{R} & =\sum_{j \neq i} \sigma_{i j} w_{d}\left(r_{i j} / r_{c}^{d}\right) \theta_{i j} \boldsymbol{e}_{i j}
\end{aligned}
$$

where $a_{i j}$ is the conservative force coefficient and $r_{i j}=\left|\mathbf{r}_{i j}\right|=\left|\mathbf{r}_{i}-\mathbf{r}_{j}\right| . w$ and $w_{d}$ are weighting functions with cutoff distances of $r_{c}$ and $r_{c}^{d}$, respectively. $\boldsymbol{e}_{i j}=\mathbf{r}_{i j} / r_{i j}$, and $\boldsymbol{v}_{i j}=\mathbf{v}_{i}-\mathbf{v}_{j} . \theta_{i j}$ is a symmetric random variable with zero mean and unit variance. $\gamma_{i j}$ and $\sigma_{i j}$ are lated by the Fluctuation-Dissipation Theorem as $\gamma_{i j}=\sigma_{i j}^{2} / 2 k_{B} T$, where $k_{B}$ is the Boltzmann constant. Mass, length, and time in the above model are measured by $m, r_{c}$, and $\sqrt{k_{B} T / m}$, respectively. We assume that the unit length and time in DPD map to physical length $[\mathrm{L}]$ and time $[\mathrm{t}]$, respectively. To satisfy the proposed requirements, we enforce

$$
\begin{aligned}
d^{2} / \nu[t] & =\bar{d}^{2} / \bar{\nu} \\
\sqrt{k_{B} T / \rho d^{3}}[L] /[t] & =\sqrt{\overline{k_{B} T} / \bar{\rho} \bar{d}^{3}}
\end{aligned}
$$

Combining Equs. (556)

$$
\begin{aligned}
{[t] } & =[L]^{2} \nu / \bar{\nu} \\
k_{B} T & =\frac{\rho \nu^{2}}{\overline{\rho \nu}^{2}} \frac{k_{B} T}{[L]}
\end{aligned}
$$

Equs. (778) can be used to setup and analyze DPD simulations of fluctuating hydrodynamics. Equ. (7) provides the mapping of time scale in DPD model. Equ. (8) indicates that the temperature in DPD simulation of fluctuating hydrodynamics is determined by the level of coarse-graining (represented by $[\mathrm{L}]$ and $\rho$ ) and transport properties of DPD fluids (represented by $\nu$ ). If $\nu$ and $\rho$ are known, then the system temperature is uniquely determined. In practice, since $\nu$ of DPD fluids is a function of their temperature, the temperature of DPD system can only be determined after the dependence of $\nu$ on temperature is known. While Equs. (5) and (6) have been used to map DPD models to physical scales, enforcing them simultaneously in fluctuating hydrodynamics simulations and thus leading to a unique choice of system temperature has not been proposed.

To demonstrate the above mapping scheme and to investigate the ability of DPD in modeling fluctuating hydrodynamics, we study the diffusion of a single nanoparticle immersed in fluids with $\bar{\rho}=10^{3}$ $\mathrm{kg} / \mathrm{m}^{3}$ and $\bar{\nu}=0.89 \times 10^{-6} \mathrm{~m}^{2} / \mathrm{s}$ at $300 \mathrm{~K}$. We set $[\mathrm{L}]=10 \mathrm{~nm}$ and $\rho=6.0$. The nanoparticle is built by bonding 117 DPD beads together and is modeled as a rigid body. In Equ. (21), $w\left(r_{i j} / r_{c}\right)=1-r_{i j} / r_{c}$ with $r_{c}=1.0$ and $a_{i j}$ is set to $a_{f f}=10.0$ and $a_{f p}=$ 17.0, where $f f$ and $f p$ denote fluid-fluid and fluidnanoparticle interactions, respectively. In Equ. (3), $w_{d}\left(r_{i j} / r_{c}^{d}\right)=\sqrt[4]{1-r_{i j} / r_{c}^{d}}$ suggested in Ref. 12 is used with $r_{c}^{d}=1.0 . \sigma_{i j}$ in Equ. (4) is taken as 5.0 for all bead pairs. Using these parameters, we first measured the viscosity of DPD fluids as a function of temperature, and the temperature in DPD model of the nanoparticle-fluids system is then determined via Equ. (8) to be 0.3875 . To investigate the ability of DPD model in reproducing the macroscopic effects of thermal fluctuations, we computed the diffusion coefficient $\left(D_{p}\right)$ of the nanoparticle, which is a macroscopic manifest of the thermal fluctuations. Fig. 1(a) shows the mean square displacement of the particle, and a $D_{p}$ of $(1.59 \pm 0.12) \times 10^{-4}$ was obtained. We also computed $D_{p}$ independently by using the Einstein-Stokes law $D_{p}=k_{B} T / 6 \pi R \mu$, where $\mu$ is the fluid viscosity. From the particlefluids pair correlation function shown in Fig. 1(b), the nanoparticle radius $R$ was determined to be 1.65 , with which a $D_{p}$ of $1.55 \times 10^{-4}$ was then computed. Given the ambiguity in the definition of particle diameter and the statistical uncertainty of DPD results, the agreement between DPD simulation and

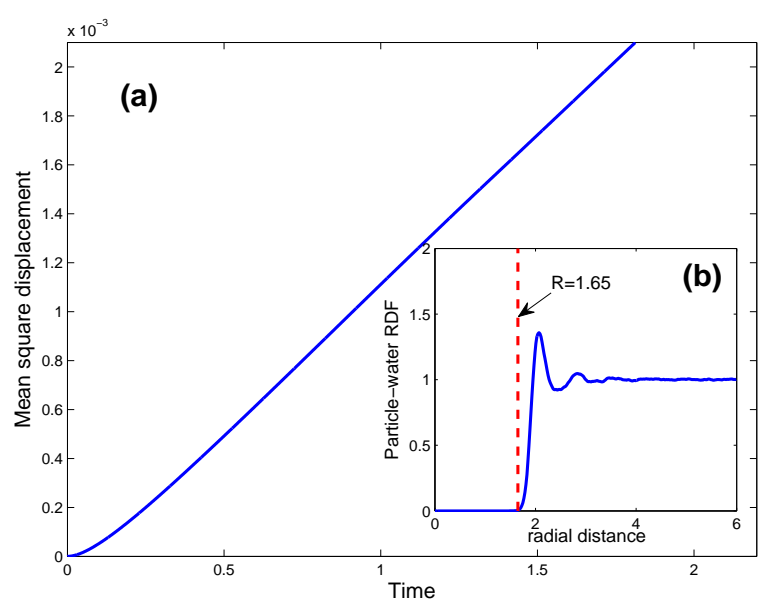

FIG. 1: (a). Mean square displacement of the nanoparticle immersed in fluids, (b) Particle-fluid pair correlation obtained from DPD simulation. 
Einstein-Stokes prediction is very good. This verifies the ability of DPD in capturing the macroscopic effects of thermal fluctuations in particulate suspensions, which has been assumed but not explicitly confirmed in the literature.

In summary, we proposed a method of mapping DPD simulation of fluctuating hydrodynamics, and the temperature in such simulations is determined uniquely by the level of coarse-graining and properties of DPD fluids. Following the proposed method of choosing system temperature, we studied the Brownian diffusion of a nanoparticle and showed that DPD simulation can reproduce the macroscopic fluctuation of nanoparticle immersed in fluids with good accuracy.
1 P. J. Hoogerbrugge and J. M. V. A. Koelman, EuroPhys. Lett. 19, 155 (1992).

2 P. Espanol and P. Warren, Europhys. Lett. 30, 191 (1995).

3 R. D. Groot and P. B. Warren, J. Chem. Phys. 107, 4423 (1997).

4 J. T. Padding and A. A. Louis, Phys. Rev. E 74, 031402 (2006).

5 R. D. Groot and K. L. Rabone, Biophys. J. 81, 725 (2001).

6 V. Symeonidis, G. E. Karniadakis, and B. Caswell, Phys. Rev. Lett. 95, 076001 (2005).
7 P. D. Palma, P. Valentini, and M. Napolitano, Phys. Fluids 18, 027103 (2006).

8 R. Prasher, P. Bhattacharya, and P. Phelan, Phys. Rev. Lett. 94, 025901 (2005).

${ }^{9}$ R. Qiao and P. He, Molecular Simu. 33, 677 (2007).

${ }^{10}$ L. D. Landau and E. M. Lifshitz, Fluid Mechanics (Pergamon Press, Oxford, 1959).

11 N. Sharma and N. A. Patankar, J. Comput. Phys. 201, 466 (2004).

12 X. Fan, N. Phan-Thien, S. Chen, X. Wu, and T. Y. Ng, Phys. Fluids 18, 063102 (2006). 